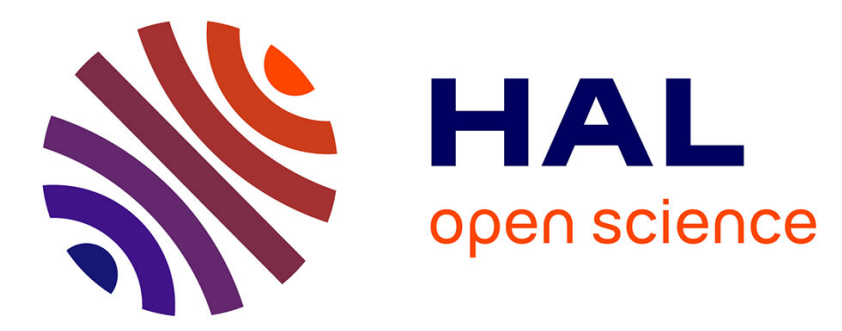

\title{
Monte Carlo Estimates of Domain-Deformation Sensitivities
}

Maxime Roger, Stéphane Blanco, Mouna El-Hafi, Richard A Fournier

\section{To cite this version:}

Maxime Roger, Stéphane Blanco, Mouna El-Hafi, Richard A Fournier. Monte Carlo Estimates of Domain-Deformation Sensitivities. Physical Review Letters, 2005, 95 (18), 180601 - 4 p. 10.1103/PhysRevLett.95.180601 . hal-01736284

\section{HAL Id: hal-01736284 https://imt-mines-albi.hal.science/hal-01736284}

Submitted on 16 Mar 2018

HAL is a multi-disciplinary open access archive for the deposit and dissemination of scientific research documents, whether they are published or not. The documents may come from teaching and research institutions in France or abroad, or from public or private research centers.
L'archive ouverte pluridisciplinaire $\mathbf{H A L}$, est destinée au dépôt et à la diffusion de documents scientifiques de niveau recherche, publiés ou non, émanant des établissements d'enseignement et de recherche français ou étrangers, des laboratoires publics ou privés. 


\title{
Monte Carlo Estimates of Domain-Deformation Sensitivities
}

\author{
Maxime Roger, ${ }^{1,2}$ Stephane Blanco,${ }^{2}$ Mouna El Hafi, ${ }^{1}$ and Richard Fournier ${ }^{2}$ \\ ${ }^{1}$ Ecole des Mines d'Albi-Carmaux, UMR CNRS 2392, 31 allées des Sciences - 81000 Albi Cedex 09, France \\ ${ }^{2}$ Laboratoire d'Energétique, Université Paul Sabatier Toulouse 3, 118 route de Narbonne, 31062 Toulouse Cedex 4, France
}

(Received 12 April 2005; published 24 October 2005)

\begin{abstract}
It is shown that, when a Monte Carlo algorithm is used for estimation of any physical quantity $A$, a simple and fast additional procedure can be implemented that simultaneously estimates the sensitivity of $A$ to any problem parameter. The proposed approach is general and systematic in the sense that: (i) it includes domain-deformation sensitivities, i.e., cases where a change in the parameter modifies the domain over which the sampled random variables are defined and (ii) a simple generic procedure is presented to address all remaining free choices in terms of variance minimization.
\end{abstract}

DOI: 10.1103/PhysRevLett.95.180601

Monte Carlo methods provide powerful tools for a wide range of problems in statistical physics, biology, engineering science, and medical applications [1,2]. In particular, an important field of applications for Monte Carlo methods is classical particle transport, where it commonly serves as reference for all other numerical approaches, and remains the only practical method as soon as either the collision operator or the system geometry is of a high level of complexity. The Monte Carlo method allows one to develop intuitive algorithms to estimate the average value $\langle O\rangle$ of an observable $O(\mathbf{x})$, simply following the probability density functions $p_{\varphi}(\mathbf{x})$ provided by the laws of the considered stochastic physical processes: $\langle O\rangle \approx \frac{1}{N} \times$ $\sum_{i=1}^{N} O\left(\mathbf{x}_{i}\right)$, where $\left\{\mathbf{x}_{i}\right\}_{i=1, N}$ is a finite set of configurations distributed according to $p_{\varphi}$. Classically, this initial point of view may be usefully combined with approaches based on integral formulations in which statistics are fully explicit and sampling probability density functions $p(\mathbf{x})$ are chosen arbitrarily:

$$
\langle O\rangle=\int_{\mathcal{D}} p_{\varphi}(\mathbf{x}) O(\mathbf{x}) d \mathbf{x}=\int_{\mathcal{D}} p(\mathbf{y}) d \mathbf{y} \frac{p_{\varphi}(\mathbf{y}) O(\mathbf{y})}{p(\mathbf{y})}
$$

and

$$
\langle O\rangle \approx \frac{1}{N} \sum_{i=1}^{N} \frac{p_{\varphi}\left(\mathbf{y}_{i}\right) O\left(\mathbf{y}_{i}\right)}{p\left(\mathbf{y}_{i}\right)}
$$

where $\mathcal{D}$ is the integration domain for $\mathbf{x}$ and $\left\{\mathbf{y}_{i}\right\}_{i=1, N}$ is a finite set of configurations distributed according to $p$. In particular, this allows one to benefit from all formalisms developed over the years for variance reduction [integral reformulation, importance sampling, and stratified sampling $[3,4]]$ that improve very significantly the accuracy of the method.

In the very same manner, it can be easily observed that integral formulations can offer the opportunity to extend Monte Carlo algorithms in such a way that, at the same time that $\langle O\rangle$ is evaluated, its sensitivity $\partial_{\lambda}\langle O\rangle$ to any parameter $\lambda$ is also evaluated at the price of a low supplementary computation effort $[5,6]$. Indeed, differentiation
PACS numbers: 05.10.Ln, 02.70.Tt, 05.60.-k

under the integral in Eq. (1) leads to

$$
\partial_{\lambda}\langle O\rangle=\int_{\mathcal{D}} p(\mathbf{y}) \frac{p_{\varphi}(\mathbf{y})}{p(\mathbf{y})}\left[\partial_{\lambda} O(\mathbf{y})+O(\mathbf{y}) \frac{\partial_{\lambda} p_{\varphi}(\mathbf{y})}{p_{\varphi}(\mathbf{y})}\right] d \mathbf{y}
$$

and

$$
\partial_{\lambda}\langle O\rangle \approx \frac{1}{N} \sum_{i=1}^{N} \frac{p_{\varphi}\left(\mathbf{y}_{i}\right)}{p\left(\mathbf{y}_{i}\right)}\left[\partial_{\lambda} O\left(\mathbf{y}_{i}\right)+O\left(\mathbf{y}_{i}\right) \frac{\partial_{\lambda} p_{\varphi}\left(\mathbf{y}_{i}\right)}{p_{\varphi}\left(\mathbf{y}_{i}\right)}\right] .
$$

In the direct relation between Eqs. (1) and (3) the only point to be highlighted is that it is always possible to retain a formulation for the sensitivity that preserves the probability density function chosen for the integral evaluation. This is the reason why the supplementary computational effort is low: the same configuration $\left\{\mathbf{y}_{i}\right\}_{i=1, N}$ is used for the evaluation of both $\langle O\rangle$ and its sensitivity, and therefore no additional random sampling is required. Altogether, this means that when one is deriving a new Monte Carlo method to address any average observable, the sensitivities of this average to all physical parameters can be simultaneously addressed. This also means that any existing Monte Carlo method can be simply extended to address sensitivities, provided that the corresponding integral formulations are available (which is the case for all advanced Monte Carlo approaches as integral formulations are necessarily made explicit when implementing variance reduction techniques).

However, a strong restriction remains: differentiation under the integral leading to Eq. (3) is only valid if the parameter under consideration does not affect the integration domain $\mathcal{D}$. In [6], the authors presented radiation transport illustrations, addressing sensitivities of a given average observable to the constants of the collision operator (mean free path, asymmetry parameter of the phase function). Indeed, the collision operator does not affect the space of all possible particle trajectories; it only affects their statistical distribution. However, if one wants to address sensitivities to the parameters defining the geometry of the system, the situation is more complex because these parameters modify the integration domain: if the geometry changes, some trajectories disappear or appear as they are 
blocked or allowed by the boundary deformation. And yet the need for geometric sensitivities is easy to identify for all types of design purposes in engineering sciences, for shielding problems in nuclear sciences, image processing, and rendering in computer graphics, etc. This need is particularly obvious nowadays in the field of medical particle imaging where one commonly faces requirements of fast and accurate computations of signal sensitivities to geometric parameters, for instance, to the locations and sizes of malignant tissues or to the locations and angles of receptors and sources.

In this Letter, we propose an effective solution to bypass the above mentioned restriction. A domain-deformation velocity field is introduced to move the arising integration difficulty from the domain boundary to the domain itself, which again allows the use of the same set of configurations. After a theoretical part describing the principle of the method, we concentrate on two academic examples of radiation transport. In order to first comment on what we mean by "domain" and "domain deformation," let us start by a brief presentation of these two examples. In the first example a one-dimensional slab of thickness $h$, filled with a uniform absorbing gas of absorption coefficient $k_{a}$, is submitted to a uniform and isotropic radiative intensity $I$ on one of its faces (of normal $\mathbf{n}$ ) and the quantity we consider is the radiative power absorbed by the gas [in the form of Eq. (1)]:

$$
P_{a}=\int_{\nu_{1}}^{\nu_{2}} p_{N}(\nu) d \nu \int_{H} p_{U}(\mathbf{u}) d \mathbf{u} \frac{(\mathbf{u} \cdot \mathbf{n}) I(\nu)\left(1-e^{-k_{a}(\nu) d_{0}}\right)}{p_{N}(\nu) p_{U}(\mathbf{u})},
$$

where $d_{0}=\frac{h}{\mathbf{u} \cdot \mathbf{n}}, p_{N}$ is an arbitrary probability density function for the frequency $\nu$ within the spectral interval $\left[\nu_{1}, \nu_{2}\right]$, and $p_{U}$ is an arbitrary probability density function for the incident direction $\mathbf{u}$ within the unit hemisphere $H$. The integration domain is therefore $\mathcal{D}=\left[\nu_{1}, \nu_{2}\right] \times H$. Example 1 is the sensitivity of $P_{a}$ to the deformation of the spectral interval (sensitivity to the spectral resolution of a given receptor). This is therefore an example of a domain deformation that has nothing to do with geometric deformation. The second illustration example (example 2) is strictly identical to example 1, except that the gas is now both absorbing and scattering (scattering coefficient $k_{s}$ ). This distinction modifies significantly the complexity of the integration domain that is now of infinite dimension because of multiple scattering:

$$
\begin{aligned}
P_{a}= & \int_{\nu_{1}}^{\nu_{2}} p_{N}(\nu) d \nu \int_{H} p_{U}(\mathbf{u}) d \mathbf{u} \frac{(\mathbf{u} \cdot \mathbf{n}) I(\nu)}{p_{N}(\nu) p_{U}(\mathbf{u})} \\
& \times\left\{e^{-k_{s}(\nu) d_{0}}\left(1-e^{-k_{a}(\nu) d_{0}}\right)+\int_{0}^{d_{0}} p_{\Sigma_{1}}\left(\sigma_{1}\right) d \sigma_{1} \int_{S} p_{U_{1}}\left(\mathbf{u}_{1}\right) d \mathbf{u}_{1} \frac{k_{s}(\nu) e^{-k_{s}(\nu) \sigma_{1}} \phi\left(\mathbf{u}_{1} \mid \mathbf{u}\right)}{p_{\Sigma_{1}}\left(\sigma_{1}\right) p_{U_{1}}\left(\mathbf{u}_{1}\right)}\right. \\
& \left.\times\left\{e^{-k_{s}(\nu) d_{1}}\left(1-e^{-k_{a}(\nu)\left(\sigma_{1}+d_{1}\right)}\right)+\int_{0}^{d_{1}} p_{\Sigma_{2}}\left(\sigma_{2}\right) d \sigma_{2} \int_{S} p_{U_{2}}\left(\mathbf{u}_{2}\right) d \mathbf{u}_{2} \ldots\right\}\right\},
\end{aligned}
$$

where $p_{\Sigma_{i}}$ is an arbitrary probability density function for the position of the $i$ th scattering event within the segment of all possible scattering positions (of length $d_{i-1}$ ), as seen from the $(i-1)$ th scattering location, in the $(i-1)$ th scattering direction $\mathbf{u}_{i-1}$. The scattering phase function (the probability density function of the scattering direction knowing the incident direction) is $\phi$ and $p_{U_{i}}$ is an arbitrary probability density function for the $i$ th scattering direction $\mathbf{u}_{i}$ in the unit sphere $S$. The integration domain is therefore: $\mathcal{D}=\mathcal{D}_{0} U \mathcal{D}_{1} U \mathcal{D}_{2} U \ldots$, where $\mathcal{D}_{0}$ coincides with the integration domain identified in example 1 and where for $i \geq 1: \mathcal{D}_{i}=\left[\nu_{1}, \nu_{2}\right] \times H \times \prod_{j=0}^{i-1}\left(S \times\left[0, d_{j}\right]\right)$. The sensitivity problem considered for illustration will here be related to a geometric deformation: we will address the sensitivity of $P_{a}$ to the slab thickness (that affects all subdomains for $i \geq 1$ ). To summarize: example 1 shows how domain deformations can be encountered although no change in geometry is considered and example 2 shows that when changes in geometry are considered, the relevant domain deformation is that of the Monte Carlo integration domain (here of infinite dimension whereas the geometry is one dimensional).

Theory. - Starting from Eq. (1), we now address the sensitivity of $\langle O\rangle$ to a parameter $\lambda$ in the general case where the integration domain $\mathcal{D}$ depends on $\lambda$. It is assumed that in the vicinity of the parameter value, the evolution of $\mathcal{D}$ with $\lambda$ is differentiable and keeps its topology unchanged. It is further assumed that the domain boundary $\partial \mathcal{D}$ is a piecewise smooth hypersurface. Under these assumptions there exists an infinite number of $C_{1}$ transformation velocity fields that match the given domain deformation (Fig. 1). On the basis of Eq. (3), choosing one among these velocity fields, $\mathbf{V}_{\lambda}$, permutation of the differentiation and integration operators is simply corrected by an integration over $\partial \mathcal{D}$ of the domain deformation rate:

$$
\begin{aligned}
\partial_{\lambda}\langle O\rangle= & \int_{\mathcal{D}} p(\mathbf{y}) \frac{p_{\varphi}(\mathbf{y})}{p(\mathbf{y})}\left[\partial_{\lambda} O(\mathbf{y})+O(\mathbf{y}) \frac{\partial_{\lambda} p_{\varphi}(\mathbf{y})}{p_{\varphi}(\mathbf{y})}\right] d \mathbf{y} \\
& +\int_{\partial \mathcal{D}} p_{\varphi}(\mathbf{z}) O(\mathbf{z}) \mathbf{V}_{\lambda}(\mathbf{z}) \cdot \mathbf{n}(\mathbf{z}) d \mathbf{z},
\end{aligned}
$$

where $\mathbf{n}(\mathbf{z})$ is the outward normal unit vector at location $\mathbf{z}$. Estimating $\partial_{\lambda}\langle O\rangle$ with Monte Carlo according to this formulation would require that a specific Monte Carlo algorithm be developed for the integral over $\partial \mathcal{D}$. This is of no interest in the present context where one of the objectives is to introduce minor algorithmic modifications into existing Monte Carlo codes in order to additionally estimate 


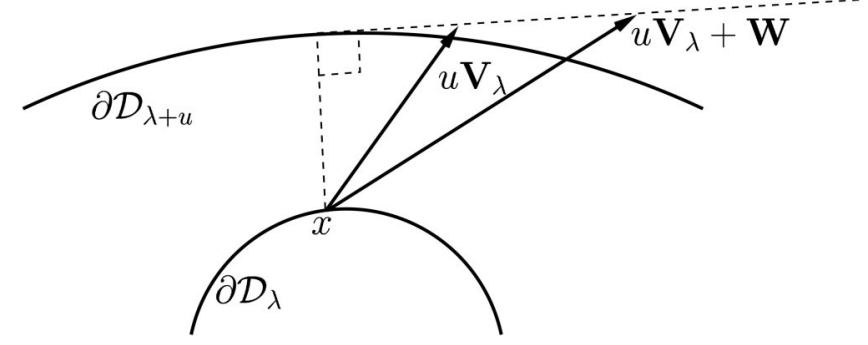

FIG. 1. The domain $\mathcal{D}$ in $\mathbb{R}^{n}$ can always be defined as $\{\mathbf{x} \in$ $\left.\mathbb{R}^{n} \mid f(\mathbf{x}, \lambda)<0\right\}$, where $f: \mathbb{R}^{n} \times \mathbb{R} \rightarrow \mathbb{R}$ is $C_{1}$ in $\lambda$. $\mathbf{V}_{\lambda}$ is constrained by $\left.\frac{d}{d u} f\left(\mathbf{x}+u \mathbf{V}_{\lambda}, \lambda+u\right)\right|_{u=0}=0$ which only fixes the normal component at the boundary $\partial \mathcal{D}$ [8]. If $\mathbf{W}$ is any vector field tangential to $\partial \mathcal{D}, \mathbf{V}_{\lambda}+\mathbf{W}$ is also solution, which means that there is a large freedom of choice for the transformation velocity field.

domain-deformation sensitivities. However, the integral over the boundary can be simply transformed into an integral over $\mathcal{D}$ by use of the Gauss-Green-Ostrogradsky theorem:

$$
\begin{aligned}
\partial_{\lambda}\langle O\rangle= & \int_{\mathcal{D}} p(\mathbf{y}) \frac{p_{\varphi}(\mathbf{y})}{p(\mathbf{y})}\left(\partial_{\lambda} O(\mathbf{y})+O(\mathbf{y}) \frac{\partial_{\lambda} p_{\varphi}(\mathbf{y})}{p_{\varphi}(\mathbf{y})}\right. \\
& \left.+\frac{\left.\nabla \cdot\left(p_{\varphi} O \mathbf{V}_{\lambda}\right)\right|_{\mathbf{y}}}{p_{\varphi}(\mathbf{y})}\right) d \mathbf{y} .
\end{aligned}
$$

The operator $\nabla$ has the dimension of $\mathcal{D}$ and is very distinct, in most cases, from that of the geometric space. With Eq. (8) we are back to the technique proposed in [6], the remaining question being the choice of the deformation velocity field $\mathbf{V}_{\lambda}$. This choice can be performed an infinite number of ways and will have no effect on the expectation value of the estimate. Only the variance will be affected. We therefore have complete freedom for optimization of statistical convergence: in the same way as the sampling probability density function $p$ could be chosen arbitrarily for optimum estimation of $\langle O\rangle$, the deformation velocity field $\mathbf{V}_{\lambda}$ can be chosen arbitrarily for optimum estimation of $\partial_{\lambda}\langle O\rangle$. Without further investigation, a meaningful choice is to minimize the spatial variations of $\mathbf{V}_{\lambda}$ : strong local variations of $\mathbf{V}_{\lambda}$ would indeed increase the variance of the Monte Carlo estimate. One obvious solution is to first define the domain-deformation velocity at the boundary and then extend it to the entire domain according to the solution of the Dirichlet problem $\left(\Delta \mathbf{V}_{\lambda}=0\right)$. This particular extension avoids strong local variations of $\mathbf{V}_{\lambda}$ in the sense that it minimizes $\int_{\mathcal{D}}\left\|\boldsymbol{\nabla}\left(\mathbf{V}_{\lambda}\right)\right\|^{2} d \mathbf{y}$. However, this attractive solution becomes impractical when increasing the domain dimension, the numerical integration of the Laplace equation requiring computational efforts that are incompatible with the present objectives.

We propose to bypass this difficulty by rewriting the integral over $\mathcal{D}$ as a succession of $n$ one-dimensional integrals, and solving $n$ trivial one-dimensional Dirichlet problems (linear extension) for each component of the deformation velocity. This corresponds in no way to the solution on the $n$-dimensional Dirichlet problem but provides a deformation velocity field that is spatially smooth enough to ensure satisfactory Monte Carlo convergence. Equation (8) may be rewritten as:

$$
\begin{aligned}
\partial_{\lambda}\langle O\rangle= & \int_{\mathcal{D}_{1}(\lambda)} d y_{1} \int_{\mathcal{D}_{2}\left(y_{1} ; \lambda\right)} d y_{2} \ldots \int_{\mathcal{D}_{n}\left(y_{1}, y_{2}, \ldots, y_{n-1} ; \lambda\right)} d y_{n} p(\mathbf{y}) \\
& \times \frac{p_{\varphi}(\mathbf{y})}{p(\mathbf{y})}\left(\partial_{\lambda} O(\mathbf{y})+O(\mathbf{y}) \frac{\partial_{\lambda} p_{\varphi}(\mathbf{y})}{p_{\varphi}(\mathbf{y})}\right. \\
& \left.+\frac{\left.\boldsymbol{\nabla} \cdot\left(p_{\varphi} O \mathbf{V}_{\lambda}\right)\right|_{\mathbf{y}}}{p_{\varphi}(\mathbf{y})}\right)
\end{aligned}
$$

where $\mathcal{D}_{1}(\lambda)$ is the space of all possible values for $y_{1}$ independent of the other coordinate values, and $\mathcal{D}_{i}\left(y_{1}, y_{2}, \ldots, y_{i-1} ; \lambda\right)$ is the space of all possible values for $y_{i}$ knowing that the $i-1$ first coordinates take the values $\left(y_{1}, y_{2}, \ldots, y_{i-1}\right)$. Each subdomain $\mathcal{D}_{i}$ is one dimensional and is the union of $m_{i}$ intervals $\left[a_{i, j}, b_{i, j}\right]$. The

TABLE I. Simulation results for $P_{a}$ and its sensitivity to $\Delta \nu$ for example 1 with $\nu_{c}=\frac{\nu_{1}+\nu_{2}}{2}$. Standard deviations are given for each Monte Carlo estimate as well as the computation time increase $\frac{t}{t_{0}}$ associated to the sensitivity calculation ( $t_{0}$ is the computation time for estimation of $A$ only and $t$ is the computation time for estimation of both $A$ and its sensitivity). The sampling probability density functions are $P_{N}(\nu)=\frac{1}{\nu_{2}-\nu_{1}}$ and $P_{U}(\mathbf{u})=\frac{\mathbf{u} \cdot \mathbf{n}}{\pi}$. The number of sampling events is $10^{6}$. The last two columns correspond to sensitivity

\begin{tabular}{|c|c|c|c|c|c|c|c|c|}
\hline$\frac{\delta}{\Delta \nu}$ & $\frac{S h}{\pi \delta}$ & $A=\frac{P_{a}}{\pi I \Delta \nu}$ & $\sigma_{A}$ & $\delta \partial_{\Delta \nu} A$ & $\delta \sigma_{\partial_{\Delta \nu} A}$ & $\frac{t}{t_{0}}$ & $\frac{t_{\text {std }}^{5 \%}}{t_{0}}$ & $\frac{p_{\text {adj }}}{\Delta \nu}$ \\
\hline 0.01 & 1 & $3.78 \times 10^{-2}$ & $1.23 \times 10^{-4}$ & $-3.71 \times 10^{-4}$ & $8.53 \times 10^{-7}$ & 1.048 & $3.33 \times 10^{3}$ & 2.04 \\
\hline 1 & 1 & 0.757 & $1.19 \times 10^{-4}$ & $-4.55 \times 10^{-2}$ & $4.27 \times 10^{-5}$ & 1.059 & $1.24 \times 10^{4}$ & 3.94 \\
\hline 10 & 1 & 0.780 & $1.10 \times 10^{-4}$ & $-4.94 \times 10^{-3}$ & $4.93 \times 10^{-6}$ & 1.075 & $7.98 \times 10^{7}$ & 316 \\
\hline 1 & 0.01 & $1.81 \times 10^{-2}$ & $2.01 \times 10^{-5}$ & $-2.43 \times 10^{-3}$ & $3.58 \times 10^{-6}$ & 1.075 & $5.04 \times 10^{4}$ & 7.94 \\
\hline 1 & 1 & 0.757 & $1.19 \times 10^{-4}$ & $-4.55 \times 10^{-2}$ & $4.27 \times 10^{-5}$ & 1.059 & $1.24 \times 10^{4}$ & 3.94 \\
\hline 1 & 10 & 0.999 & $3.92 \times 10^{-8}$ & $-4.26 \times 10^{-5}$ & $1.12 \times 10^{-7}$ & 1.071 & 196 & 0.495 \\
\hline
\end{tabular}
estimations based on two independent Monte Carlo computations: $\partial_{\Delta \nu} A \simeq \frac{A_{\Delta \nu+p}-A_{\Delta \nu}}{p}$. We impose that the same uncertainty level is reached as in column 6. In the first case, a $5 \%$ perturbation of the parameter is used $\left(\frac{p}{\Delta \nu}=0.05\right)$ and the number of sampling events is adjusted; $t_{\text {std }}^{5 \%}=2 t_{0}\left(\frac{\sqrt{2} \sigma_{A}}{p \sigma_{\partial \Delta \nu^{A}}}\right)^{2}$ is the corresponding computation time. In the second case, the number of sampling events is $10^{6}$ for each Monte Carlo computation $\left(\frac{t}{t_{0}}=2\right)$ and the adjustment is made on the amplitude of the parameter perturbation $\left(p=p_{\text {adj }}=\frac{\sqrt{2} \sigma_{A}}{\sigma_{\partial \nu_{\nu} A}}\right)$. 
TABLE II. Simulation results for $P_{a}$ and its sensitivity to $h$ for example 2 in the case of isotropic scattering. Standard deviations are given for each Monte Carlo estimate as well as the computation time increase $\frac{t}{t_{0}}$ associated to the sensitivity calculation. The sampling probability density functions are $P_{U}(\mathbf{u})=\frac{\mathbf{u} \cdot \mathbf{n}}{\pi}, P_{\Sigma_{i}}\left(\sigma_{i}\right)=\frac{k_{s} e^{-k_{s} \sigma_{i}}}{1-e^{-k_{s} d_{i-1}}}$, and $P_{U_{i}}\left(\mathbf{u}_{\mathbf{i}}\right)=\frac{1}{4 \pi}$. The number of sampling events is $10^{6}$. Units are arbitrary for $k_{a}, k_{s}$, and $h . t_{\text {std }}^{5 \%}$ and $p_{\text {adj }}$ are defined as in Table I.

\begin{tabular}{lccccccccc}
\hline \hline$k_{a}$ & $k_{s}$ & $h$ & $A=\frac{P_{a}}{\pi I \Delta \nu}$ & $\sigma_{A}$ & $\frac{1}{k_{s}+k_{a}} \partial_{h} A$ & $\frac{1}{k_{s}+k_{a}} \sigma_{\partial_{h} A}$ & $\frac{t}{t_{0}}$ & $\frac{t_{s d}^{5 / d}}{t_{0}}$ & $\frac{p_{\text {adj }}}{h}$ \\
\hline 1 & 1 & 0.001 & $1.99 \times 10^{-3}$ & $2.98 \times 10^{-6}$ & 0.991 & $2.08 \times 10^{-3}$ & 1.12 & 821 & 1.01 \\
1 & 1 & 1 & 0.748 & $2.22 \times 10^{-4}$ & 0.107 & $6.09 \times 10^{-4}$ & 1.11 & 53.2 & 0.258 \\
1 & 1 & 10 & 0.853 & $2.52 \times 10^{-4}$ & $3.43 \times 10^{-5}$ & $2.23 \times 10^{-4}$ & 1.11 & 5.11 & $7.99 \times 10^{-2}$ \\
1 & 0 & 1 & 0.780 & $1.10 \times 10^{-4}$ & 0.297 & $9.78 \times 10^{-5}$ & 1.23 & $2.02 \times 10^{3}$ & 1.59 \\
1 & 1 & 1 & 0.748 & $2.22 \times 10^{-4}$ & 0.107 & $6.09 \times 10^{-4}$ & 1.11 & 53.2 & 0.258 \\
1 & 10 & 1 & 0.501 & $3.62 \times 10^{-4}$ & $1.45 \times 10^{-3}$ & $3.79 \times 10^{-4}$ & 1.089 & 12.1 & 0.123 \\
0.001 & 1 & 1 & $1.99 \times 10^{-3}$ & $1.54 \times 10^{-6}$ & $1.99 \times 10^{-3}$ & $5.80 \times 10^{-6}$ & 1.11 & 113 & 0.375 \\
1 & 1 & 1 & 0.748 & $2.22 \times 10^{-4}$ & 0.107 & $6.09 \times 10^{-4}$ & 1.11 & 53.2 & 0.258 \\
10 & 1 & 1 & 0.980 & $9.77 \times 10^{-5}$ & $-7.89 \times 10^{-5}$ & $1.28 \times 10^{-4}$ & 1.087 & 7.70 & $9.81 \times 10^{-2}$ \\
\hline \hline
\end{tabular}

deformation velocity is built component by component starting with $V_{\lambda, 1}$ that is a function of $y_{1}$ only. For $y_{i} \in$ $\left[a_{i, j}, b_{i, j}\right]$ :

$$
\begin{gathered}
V_{\lambda, 1}\left(y_{1} ; \lambda\right)=\partial_{\lambda} a_{1, j}+\frac{\partial_{\lambda} b_{1, j}-\partial_{\lambda} a_{1, j}}{b_{1, j}-a_{1, j}}\left(y_{1}-a_{1, j}\right) \\
V_{\lambda, i}\left(y_{1}, y_{2}, \ldots, y_{i} ; \lambda\right)=\partial_{\lambda} a_{i, j}+\frac{\left.V_{\lambda, i}\right|_{y_{i}=b_{i, j}}-\left.V_{\lambda, i}\right|_{y_{i}=a_{i, j}}}{b_{i, j}-a_{i, j}} \\
\times\left(y_{i}-a_{i, j}\right)
\end{gathered}
$$

with

$$
\begin{aligned}
\left.V_{\lambda, i}\right|_{y_{i}=a_{i, j}} & \equiv V_{\lambda, i}\left(y_{1}, y_{2}, \ldots, y_{i-1}, y_{i}=a_{i, j} ; \lambda\right) \\
& =\partial_{\lambda} a_{i, j}+\sum_{k=1}^{i-1} V_{\lambda, k}\left(y_{1}, y_{2}, \ldots, y_{k} ; \lambda\right) \partial_{y k} a_{i, j} .
\end{aligned}
$$

Practical implementation. - This systematic procedure is applied hereafter to the above described examples. For example 1, a gray incident intensity is supposed and the absorption spectrum is that of a single Lorentzian line: $k_{a}=\frac{S \delta}{\pi\left[\left(\nu-\nu_{c}\right)^{2}+\delta^{2}\right]}$. The sensitivity in question is the sensitivity to the width of the observational spectral interval, $\Delta \nu=\nu_{2}-\nu_{1}$. Table I displays the sensitivity estimation results for various values of the line intensity and the line width. In this first example, the domain is two dimensional and the implementation is trivial, but when dealing with high-dimensional spaces, determining the domains $\mathcal{D}_{i}$ in Eq. (9) may seem an insurmountable problem. Fortunately, for existing Monte Carlo algorithms, this problem vanishes as it is directly related to the generation of configuration sets in Eq. (2). The practical implementation of Monte Carlo random sampling of $\mathbf{x}_{i}$ and $\mathbf{y}_{i}$ in $\mathcal{D}$ is usually accomplished by a reduction to a succession of dependant random sampling events in low-dimensional domains following stochastic physical models. This point is illustrated with example 2 in which the domain of infinite dimension is the combination of successive one-dimensional spatial domains and two-dimensional angular domains [Eq. (6)].
In Table II, we consider a gray medium and a gray incident intensity and we evaluate the sensitivity to the slab thickness $h$. The corresponding Monte Carlo estimates are displayed for various values of $k_{a}, k_{s}$, and $h$. All sensitivities are accurately estimated in both examples for all configurations, except for the relative standard deviations which are large when the sensitivity value tends to zero. For comparison, in both tables we also report results corresponding to standard numerical differentiations. In all cases, either the required computation times are very much higher than those corresponding to the methodology presented here, or the amplitude of the required perturbation is large (raising concerns about the linearity assumption).

We are presently making use of this methodology for a wide range of radiative transfer applications, as well as for the analysis of residence times of stochastic motions in bounded domains, in particular, for the analysis of the successive moments and of their sensitivities to the parameters of the motion as well as to the geometry of the system [7].

The authors thank Jean-Marc Schlenker and Leanne Pitchford for helpful discussions.

[1] J. M. Hammersley and D. C. Handscomb, Monte Carlo Methods (Chapman and Hall, London, 1964).

[2] G.S. Fishman, Monte Carlo Concepts, Algorithms and Applications (Springer Verlag, Berlin, 1996).

[3] R. Assaraf and M. Caffarel, Phys. Rev. Lett. 83, 4682 (1999).

[4] G. A. Mikhailov, Optimization of Weighted Monte Carlo Methods (Springer-Verlag, Berlin, 1995).

[5] K. Weise and H. Zhang, J. Phys. A 30, 5971 (1997).

[6] A. De Lataillade, S. Blanco, Y. Clergent, J.-L. Dufresne, M. El hafi, and R. Fournier, J. Quant. Spectrosc. Radiat. Transfer 75, 529 (2002).

[7] S. Blanco and R. Fournier, Europhys. Lett. 61, 168 (2003).

[8] M. Spivak, A Comprehensive Introduction to Differential Geometry (Publish or Perish, Inc., Wilmington, Delaware, 1979), Vol. IV, 2nd ed. 